\title{
Editorial
}

\section{Artificial Intelligence and Data Mining 2014}

\author{
Fuding Xie, ${ }^{1}$ Suohai Fan, ${ }^{2}$ Jianzhou Wang, ${ }^{3}$ Helen $\mathbf{L u},{ }^{4}$ and Caihong $\mathrm{Li}^{5}$ \\ ${ }^{1}$ School of Urban and Environmental Sciences, Liaoning Normal University, Dalian 116029, China \\ ${ }^{2}$ School of Information Science and Technology, Jinan University, Guangzhou 510632, China \\ ${ }^{3}$ School of Mathematics and Statistics, Lanzhou University, Lanzhou 730000, China \\ ${ }^{4}$ Department of Software Engineering, University of Technology, Sydney, NSW 2007, Australia \\ ${ }^{5}$ School of Information Science and Engineering, Lanzhou University, Lanzhou 730000, China \\ Correspondence should be addressed to Fuding Xie; xiefd@lnnu.edu.cn
}

Received 21 August 2014; Accepted 21 August 2014; Published 14 October 2014

Copyright (C) 2014 Fuding Xie et al. This is an open access article distributed under the Creative Commons Attribution License, which permits unrestricted use, distribution, and reproduction in any medium, provided the original work is properly cited.

Artificial intelligence and data mining techniques have been widely used in many domains to solve classification, planning, diagnosis, computation, prediction, and optimization problems. The aim of this special issue is to reflect the latest development in this research field and provide advanced knowledge for researchers actively working on algorithms and applications of artificial intelligence. The accepted papers in this issue are concerned with the following topics: (i) forecasting models based on statistical methods and artificial intelligence; (ii) advanced artificial intelligence algorithm and novel data mining techniques; (iii) computational intelligence in medical science and biology; (iv) time series analysis in economics and finance; (v) machine learning on massive datasets.

Among them, there are nine papers regarding forecasting models based on artificial intelligence and data mining techniques. In "Short-term wind speed forecasting using decomposition-based neural networks combining abnormal detection method," authored by X. Chen et al., two threestage hybrid approaches are developed for short-term wind speed forecasting. In "Wind power assessment based on a WRF wind simulation with developed power curve modeling methods," authored by Z. Guo and X. Xiao, the authors propose two improved power curve modeling methods. In "A hybrid approach by integrating brain storm optimization algorithm with grey neural network for stock index forecasting," authored by Y. Sun, a novel hybrid model based on the brain storm optimization approach is constructed for stock index forecast. In "Hybrid wind speed forecasting model study based on SSA and intelligent optimized algorithm," authored by W. Zhang et al., the authors investigate singular spectrum analysis in three time series models to forecast wind speed. In "Intelligent optimized combined model based on GARCH and SVM for forecasting electricity price of New South Wales, Australia," authored by Y. Yang et al., the researchers suggest an optimized combined forecasting model by ant colony optimization algorithm. In "A hybrid forecasting model based on bivariate division and a backpropagation artificial neural network optimized by chaos particle swarm optimization for day-ahead electricity price," authored by Z. Wang et al., a bivariate division backpropagation artificial neural network method based on chaos particle swarm optimization is utilized for electricity price prediction. In "An optimized forecasting approach based on grey theory and cuckoo search algorithm: a case study for electricity consumption in New South Wales," authored by P. Jiang et al., the authors build a hybrid grey model optimized by cuckoo search algorithm. In "Radial basis function neural network based on an improved exponential decreasing inertia weight-particle swarm optimization algorithm for AQI prediction," authored by J. Lu et al., a novel radial basis function neural network model optimized by improved particle swarm optimization is developed. In "Deterministic echo state networks based stock price forecasting," authored by J. Dan et al., echo state network with deterministically constructed reservoir is investigated.

Second, there are six papers on new algorithm and new model design. In "Online learning discriminative dictionary with label information for robust object tracking," authored by 
B. Fan et al., a supervised approach to online learn a structured sparse and discriminative representation for object tracking is proposed. In "Approximate accuracy approaches to attribute reduction for information systems," authored by D. Liu et al., a new approach to attribute reduction for incomplete and fuzzy valued information systems is proposed. In "Moment conditions selection based on adaptive penalized empirical likelihood," authored by Y. Song, an empirical likelihood shrinkage method is developed to estimate parameters and select correct moment conditions. In " $A$ hybrid sampling SVM approach to imbalanced data classification," authored by Q. Wang, a hybrid sampling SVM approach is proposed combining oversampling and undersampling techniques. In "Cost-sensitive support vector machine using randomized dual coordinate descent method for big classimbalanced data classification," authored by M. Tang et al., a cost-sensitive support vector machine using randomized dual coordinate descent method is proposed. In "Research on adaptive optics image restoration algorithm by improved expectation maximization method," authored by L. Zhang et al., the authors put forward a deconvolution algorithm improved by the expectation maximization algorithm.

Some papers collected in this special issue are concerned with some applications to medical science and biology. In "Evaluating the risk of metabolic syndrome based on an artificial intelligence model," authored by $\mathrm{H}$. Chen et al., a model is developed to identify adults at risk of metabolic syndrome based on physical signs. In "High-risk immunization strategies for multiethnic regions," authored by F. Nian and T. Liu, some high-risk immunization strategies for multiethnic regions are proposed. In "Identification of protein coding regions in the eukaryotic DNA sequences based on Marple algorithm and wavelet packets transform," authored by G. Liu and Y. Luan, a novel algorithm based on autoregressive spectrum analysis and wavelet packets transform is presented.

Several authors are also interested in the applications to economics and finance. In "Adaptive heterogeneous autoregressive models of realized volatility based on a genetic algorithm," authored by $\mathrm{H}$. Qu and P. Ji, the authors propose an adaptive heterogeneous autoregressive model. In "Dynamic communities in stock market," authored by X. Gui et al., the authors introduce an approach to construct stock networks based on stock prices. In "A multitarget land use change simulation model based on cellular automata and its application," authored by J. Yang et al., the authors propose a multitarget land use change simulation model based on cellular automata.

Finally, other applied problems are considered on different domains. In "Comprehensive influence model of preschool children's personality development based on the Bayesian network," authored by Y. Sun et al., an influencing factor model of personality development based on the Bayesian network is proposed. In "Identifying the best coach by an improved AHP model," authored by J. Xing et al., a model is proposed to evaluate college coaches of a century. In "A weighted voting classifier based on differential evolution," authored by Y. Zhang et al., the authors propose a weighted voting approach based on differential evolution. In "The influence of traffic networks on the supply-demand balance of tourism: a case study of
Jiangsu province, China," authored by T. Chen, a road network is established to address the impact of traffic networks on the supply-demand balance of tourism. In "Recognition of process disturbances for an SPC/EPC stochastic system using support vector machine and artificial neural network approaches," authored by Y. E. Shao, the author proposes the integration of support vector machine and artificial neural network approaches to recognize the disturbance patterns. In "Target image matching algorithm based on binocular CCD ranging," authored by $\mathrm{D}$. Li et al., the authors propose target image in a subpixel level matching algorithm for binocular CCD ranging. In " $A$ solution to reconstruct cross-cut shredded text documents based on character recognition and genetic algorithm," authored by $\mathrm{H}$. Xu et al., a feature-matching algorithm based on the character recognition via establishing the database of the letters is presented.

\section{Acknowledgments}

The guest editors of this special issue would like to express their thanks to the authors who have submitted papers for consideration and the referees of the submitted papers.

Fuding Xie Suohai Fan Jianzhou Wang Helen $\mathrm{Lu}$ Caihong $\mathrm{Li}$ 


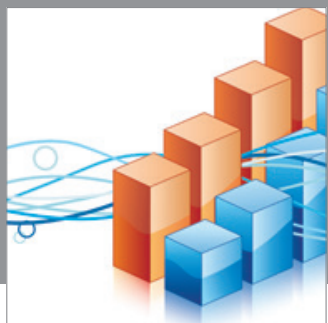

Advances in

Operations Research

mansans

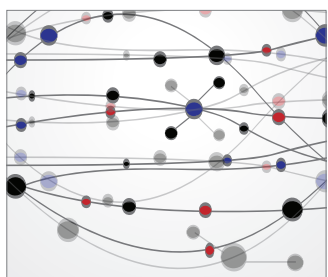

The Scientific World Journal
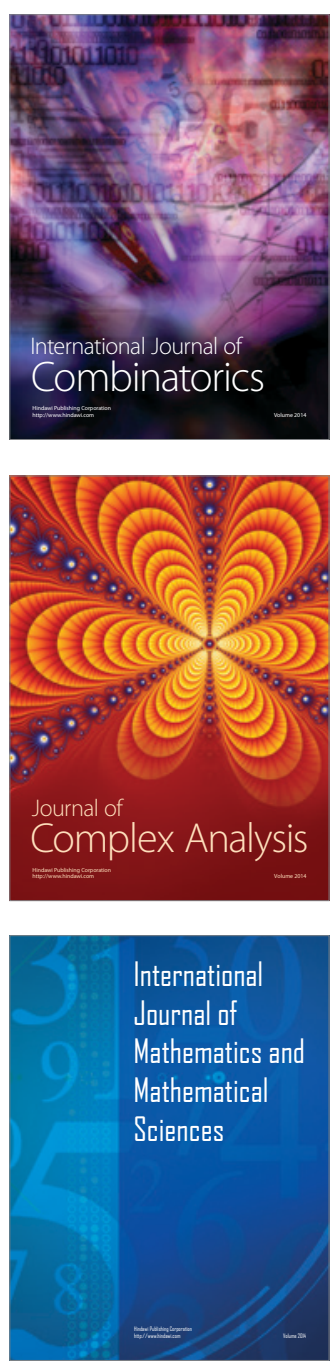
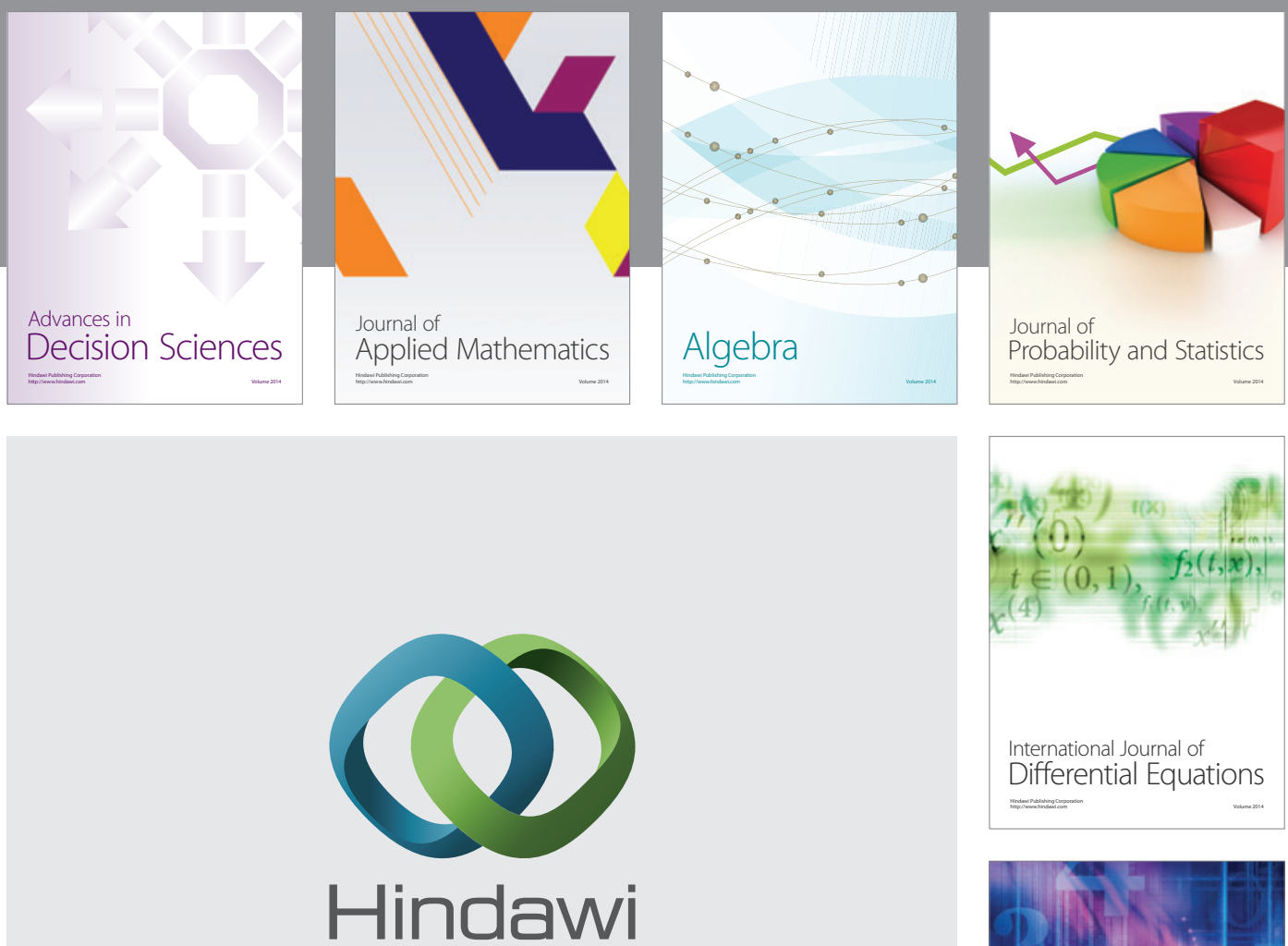

Submit your manuscripts at http://www.hindawi.com
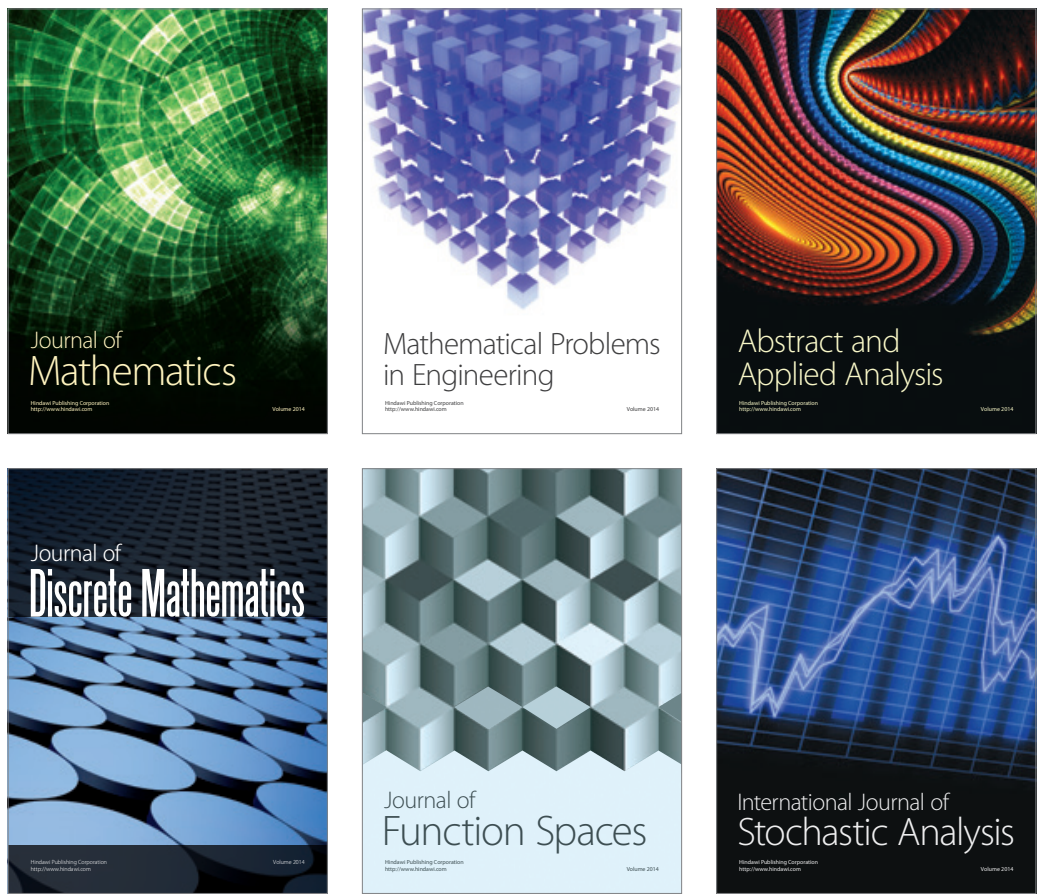

Journal of

Function Spaces

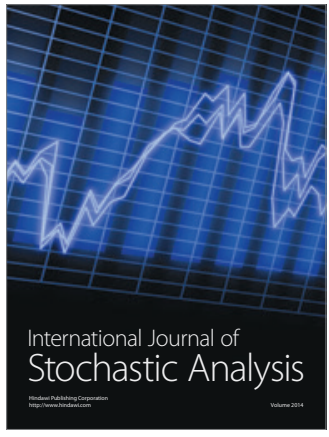

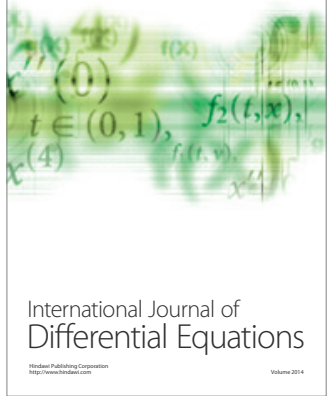
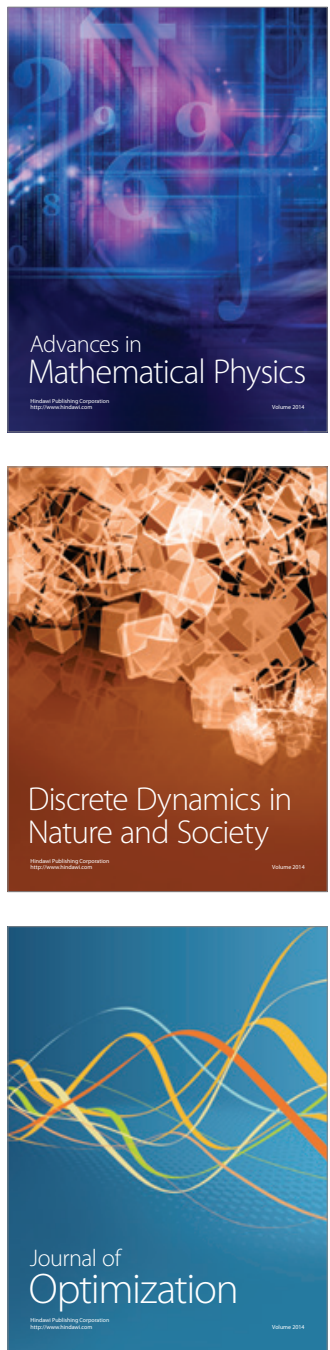